\title{
醌氧化还原酶响应型光声探针的合成及其对乳腺癌的成像
}

\author{
黄靖 ${ }^{\dagger}$ 王超 ${ }^{\dagger}$ 林敏刚曾钫* 吴水珠* \\ (华南理工大学发光材料与器件国家重点实验室 广东省分子聚集发光重点实验室 \\ 华南理工大学材料科学与工程学院 广州 510640)
}

\begin{abstract}
摘要 醌氧化还原酶(NQO1)是生物体内的一种双电子还原反应的专性催化还原酶. 在一些疾病如乳腺癌中 NQO1 过 度表达, 因此 NQO1 可作为生物标志物以观察乳腺癌肿瘤的产生和发展. 目前已有一些文献报导用于 NQO1 检测和苂 光成像的苂光探针, 但大部分这些探针具有吸收波长较短、背景干扰较强、组织穿透深度较低等不足. 为克服荧光探 针成像的上述缺陷，我们构建出一种具有推拉电子结构的可对 NQO1 响应的新型近红外光声探针 TPA-X-Q. 实验结果 表明, 该探针可在生物体内和体外对 NQO1 进行专一性的增强型光声检测和成像, 并可提供高分辨率的实时光声信号 图像. 此外, 这种新型的光声探针能够成功应用于乳腺癌小鼠模型, 对乳腺癌肿瘤中过量表达的 NQO1 进行灵敏准确 的光声检测与成像, 借助多光谱光声断层扫描技术(MSOT)的三维光声图像重建功能, 可实现肿瘤病灶区域的定位. 关键词＼cjkstart醌氧化还原酶; 探针; 近红外; 乳腺癌; 多光谱光声断层扫描
\end{abstract}

\section{Synthesis of NQO1-activatable Optoacoustic Probe and Its Imaging of Breast Cancer}

\author{
Jing Huang ${ }^{\dagger}$ Chao Wang ${ }^{\dagger}$ Mingang Lin Fang Zeng* Shuizhu Wu* \\ (South China University of Technology, State Key Laboratory of Luminescent Materials \& Devices, College of Materials \\ Science and Engineering, GuangZhou 510640, China)
}

\begin{abstract}
NAD}(\mathrm{P}) \mathrm{H}:$ quinone oxidoreductase-1 (NQO1) is a cytosolic two-electron-specific reductase whose abnormal expression is associated with breast cancer, and NQO1 has been regarded as an important biomarker for monitoring the occurrence and development of breast tumors. Although there are some research reports involving fluorescent probes for imaging NQO1 in vivo, they generally suffer from the strong light scattering by tissues which would cause the spatial resolution of fluorescent signals degrade rapidly with imaging depth. On the other hand, as an emerging detection and imaging modality, optoacoustic tomography (OAT) is capable of providing more detailed information in deep biological tissues than fluorescent imaging, since in OAT ultrasound signals are the reporting signals. To overcome the inherent defects of fluorescent imaging, in this work, we developed a novel near-infrared (NIR) NQO1-activatable optoacoustic (OA) probe TPA-X-Q based on the push-pull internal charge transfer (ICT) scaffold. TPA-X-Q consists of a NQO1-responsive moiety, quinone propionate group and a NIR chromophore TPA-X-OH. In the absence of the enzyme NQO1, the probe displays nearly no noticeable OA signal upon NIR excitation. Whereas in the presence of NQO1, the quinone propionate group of TPA-X-Q is reduced and cleaved by the enzymatic reaction triggered by NQO1, and thus TPA-X-Q is transformed into TPA-X-OH, consequently evident OA signal is generated, thereby realizing the detection and imaging of NQO1. As for the probe, the limit of detection (LOD) is determined to be $0.193 \mu \mathrm{g} \cdot \mathrm{mL}^{-1}$. Furthermore, the probe TPA-X-Q displays several advantages, such as quite good selectivity towards NQO1, low cytotoxicity and excellent photostability. Moreover, the probe has been successfully utilized to detect and image the overexpressed NQO1 in the breast cancer-bearing mouse model. Orthogonal-view three-dimensional (3D) images can also be obtained by using multispectral optoacoustic tomography (MSOT), and thus precise localization of the breast cancer tumors can be achieved. This probe holds great potential for being employed as an efficacious tool for diagnosing breast cancer via responding to NQO1.
\end{abstract}

Keywords NQO1, optoacoustic probe, near-infrared, breast cancer, MSOT

\section{1 引言}

醌氧化还原酶(NAD(P)H:quinone oxidoreductase-1,

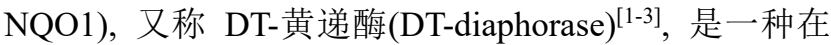
辅酶因子 NADH 或 NADPH ${ }^{[4,5]}$ 配合下能专一催化生物 体内双电子还原反应的还原酶 ${ }^{[6,7]}$. 生物体正常细胞在

*E-mail: mcfzeng@scut.edu.cn, shzhwu@scut.edu.cn; Tel.: 020-22236262; Fax: 020-22236363

$\uparrow$ 作者贡献相同.

Received October 6, 2020; published November 16, 2020.

Supporting information for this article is available free of charge via the Internet at http://sioc-journal.cn.

Project supported by the National Natural Science Foundation of China (No. 21875069), the Fund of Guangdong Provincial Key Laboratory of Luminescence from Molecular Aggregates (No. 2019B030301003), and the Natural Science Foundation of Guangdong Province (No. 2016A030312002).

项目受国家自然科学基金(No. 21875069), 广东省分子聚集发光重点实验室课题(No. 2019B030301003)和广东省自然科学基金(No. 2016A030312002)资助. 
有氧代谢的过程中, 在一定条件下可产生活性氧物质 $(\mathrm{ROS})^{[8]}$, 当 ROS 过量时会对机体组织发起攻击, 产生 氧化应激(Oxidative stress), 使细胞内的 DNA 和蛋白质 被破坏, 继而引发 DNA 突变, 造成原癌基因被激活而 抑癌基因被抑制, 形成肿瘤 ${ }^{[9-11]}$. 并且在这些癌变的细 胞中, NQO1 过量表达 ${ }^{[12-15]}$. 而且这些 NQO1 高于正常 水平的肿瘤细胞具有极强的适应能力, 可从原位瘤扩散 到机体其他部位, 形成转移瘤 ${ }^{[16]}$, 加速病情的恶化甚至 个体的死亡. 现有研究表明 [17-19], 过量表达的 NQO1 与 乳腺癌的病情发展状况密切相关. 因此 NQO1 可作为乳 腺癌的生物标志物, 应用于乳腺癌的成像和诊断中.

苂光成像技术作为一种非侵入式的检测手段, 因其 灵敏度高、可实时成像等优势已获得广泛研究 ${ }^{[20-27]}$. 目 前已有苂光探针用于生物体外或体内对 NQO1 的苂光 检测和成像方面的相关报道 ${ }^{[28-34]}$, 但是由于生物组织的 强光散射作用致使苂光信号随着成像深度的增大而急 剧衰减, 苂光成像效果下降明显. 光声断层扫描技术 (Optoacoustic tomography, OAT)作为一种新兴的成像技 术成为近年来生物成像领域的研究热点之一[35,36]. 光声 成像是基于 “光进声出” 的原理, 即以近红外脉冲激光 激发组织样品, 检测组织样品因局部热膨胀产生的超声 信号进行成像; 由于超声信号在生物组织中的散射较 低, 因此光声成像可具有较高的组织穿透深度 ${ }^{[37]}$. OAT 融合了光学成像技术和超声成像技术各自的优势, 可为 更深层的生物组织提供比传统荧光成像技术空间分辨 率更高的图像信息. 此外, 多光谱光声断层扫描技术 (Multispectral optoacoustic tomography, MSOT)利用不同 波长的近红外激发光激发样品, 检测来自组织中的不同 光吸收剂的超声信号, 通过 MSOT 系统的光谱拆分功 能, 可以区分并获取特定光吸收(如内源性或外源性的 光吸收剂)的光声信号, 并且利用三维光声成像可实现 对病灶区域的定位 ${ }^{[38-45]}$. 但是, 目前用于在生物体内外 对 NQO1 进行光声检测和成像的光声探针还未见报道.

在本文中我们设计合成了一种新型光声探针化合 物 TPA-X-Q. 通常有机小分子生色团化合物的光吸收波 长可通过引入不同的取代基团进行调节, 这是取代基对 生色团分子所产生的电子效应作用结果 ${ }^{[46-48]}$. 因此, 我 们设计并合成了基于推拉电子结构的生色团化合物 TPA-X-OH, 即在富电子的氧杂葱基团两端分别引入电 子供体三苯胺基团和电子受体吲哚盐, 形成 $D-\pi-\mathrm{A}$ 型分 子结构, 而后通过酯化偶联反应将 NQO1 的响应基团, 即醌丙酸酯基团, 偶联到生色团化合物 TPA-X-OH 上制 得光声探针 TPA-X-Q. 其可对 NQO1 进行特异性的响 应, 并由此产生较强的光声信号.

探针对 NQO1 的响应机理如图 1 所示, 探针 TPA-X-Q 在近红外区域具有较弱的近红外吸收和光声 信号强度; 而在 NQO1 的催化作用下, 醌丙酸酯基团因 酶促反应被还原、自环化并离去 ${ }^{[49]}$, 探针被激活, 生成
具有较强近红外吸收的产物一一生色团化合物 TPA-X-OH, 其光谱学性质出现明显改变, 近红外区域 的吸收 $(710 \mathrm{~nm})$ 增强. 与此同时, 光声信号增强, 因而 可实现对 NQO1 的增强型光声检测和成像. 我们通过光 谱测试验证了该探针能在生物体外对 NQO1 产生选择 性的响应. 此外, 我们还建立了雌性 BALB/c 裸鼠原位 乳腺癌模型, 并将该探针应用于该模型, 通过 MSOT 技 术实现了对生物体内肿瘤中过量表达的 NQO1 的检测 和成像.

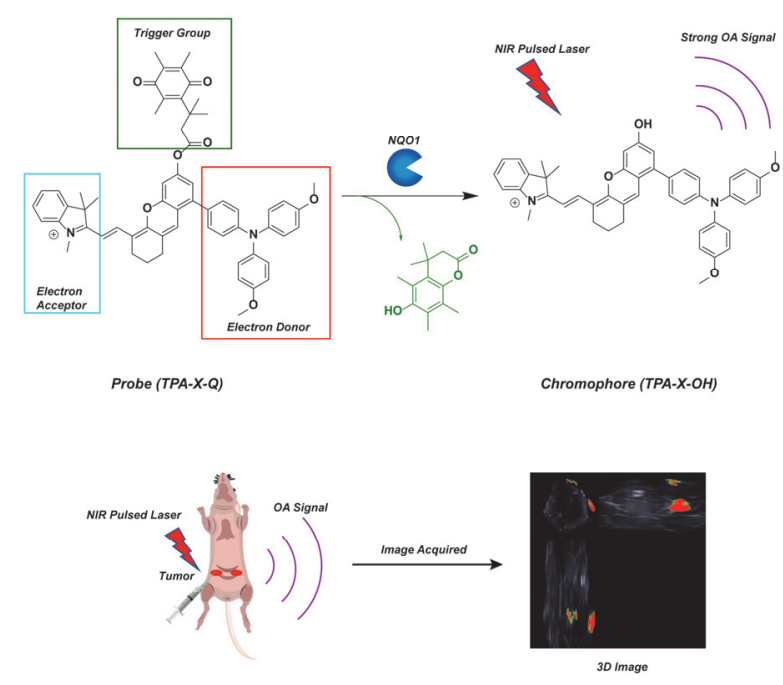

图 1 探针 TPA-X-Q 结构及其响应成像机理示意图

Figure 1 The response and imaging mechanism of TPA-X-Q

\section{2 结果与讨论}

\section{1 探针的合成与光谱性质}

生色团化合物 TPA-X-OH 和探针 TPA-X-Q 的合成 路线如图 S1 所示, 化合物的结构通过核磁共振氢谱 $\left({ }^{1} \mathrm{H}\right.$ $\mathrm{NMR}) 、$ 核磁共振碳谱 $\left({ }^{13} \mathrm{C} \mathrm{NMR}\right)$ 和高分辨质谱(HRMS) 进行了表征. 在整个分子的共轭体系内部, 供体和受体 相互作用, 从而促进分子内电荷转移(ICT), 吸收光谱移 至近红外区域. 如图 S12-13 所示, 与文献报道的生色团 化合物 $\mathrm{X}-\mathrm{OH}^{[50]}$ 相比, 引入电子供体三苯胺基团的生色 团 TPA-X-OH，由于 ICT 效应的增强，在近红外波段范 围内的吸收强度明显增加. 此外, 三苯胺基团内具有较 多可自由内旋转的碳一碳单键, 使生色团化合物吸收的 激发光能量更多地以热能的形式释放, 从而使局部热膨 胀产生的超声信号增强.

探针和生色团在 PBS 缓冲液 $(\mathrm{pH}=7.4$, 含体积分数 为 $1 \%$ 的 $\mathrm{DMSO}$ )中的吸收光谱如图 $2 \mathrm{~A}$ 所示, 生色团分 子 TPA-X-OH 的吸收峰位于 $710 \mathrm{~nm}$, 而探针分子 TPA-X-Q 吸收峰位于 $610 \mathrm{~nm}$ 左右, 这可能是由于氧杂 蒽基团上供电性的羟基基团被缺电性的醌丙酸酯基团 所取代, 分子内电荷转移效应减弱, 因此相对于生色团 化合物, 探针的吸收光谱明显蓝移, 最大吸收波长由原 
先的 $710 \mathrm{~nm}$ 变成 $610 \mathrm{~nm}$. 并且在 TPA-X-OH 的吸收峰 $710 \mathrm{~nm}$ 处, 生色团化合物的吸收强度是探针分子的 6 倍, 表明该探针具有对 NQO1 进行增强型光声检测的可 能性.
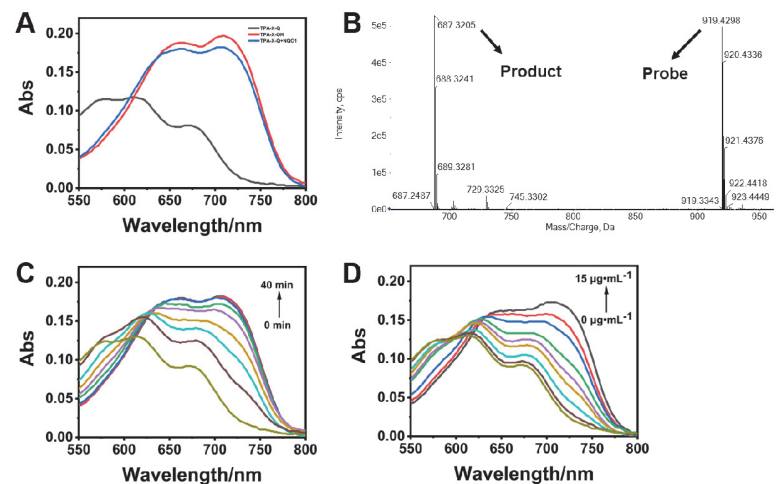

图 2 (A) 探针 TPA-X-Q $\left(10 \mu \mathrm{mol} \cdot \mathrm{L}^{-1}\right)$ 、生色团化合物 TPA-X-OH(10 $\mu \mathrm{mol} \cdot \mathrm{L}^{-1}$ ) 溶液、探针和 $\mathrm{NQO} 1$ 反应液(探针浓度 $10 \mu \mathrm{mol} \cdot \mathrm{L}^{-1}, \mathrm{NQO} 1$ 浓度 $15 \mu \mathrm{g} \cdot \mathrm{mL}^{-1}$, 反应时间 $40 \mathrm{~min}$ ) 的吸收光谱. (B) 探针与 NQO1 反 应液的 HRMS 图谱(探针浓度 $10 \mu \mathrm{mol} \cdot \mathrm{L}^{-1}$, NQO1 浓度 $15 \mu \mathrm{g} \cdot \mathrm{mL}^{-1}$, 反 应时间 $10 \mathrm{~min})$. (C) 探针 $\left(10 \mu \mathrm{mol} \cdot \mathrm{L}^{-1}\right)$ 在 $15 \mu \mathrm{g} \cdot \mathrm{mL}^{-1} \mathrm{NQO} 1$ 作用下, 不同响应时间 $(0 \sim 40 \mathrm{~min})$ 的吸收光谱变化. (D) 探针 $\left(10 \mu \mathrm{mol} \cdot \mathrm{L}^{-1}\right)$ 在 不同浓度 $\left(0 \sim 15 \mu \mathrm{g} \cdot \mathrm{mL}^{-1}\right) \mathrm{NQO1}$ 作用下 $40 \mathrm{~min}$ 后的吸收光谱变化. 所 有测试均在 $37{ }^{\circ} \mathrm{C}$ 下的磷酸盐缓冲液 $(\mathrm{pH}=7.4$, 含 $\operatorname{DMSO}(\varphi=1 \%)$ 和 $100 \mu \mathrm{mol} \cdot \mathrm{L}^{-1}$ 辅酶因子 $\left.\mathrm{NADH}\right)$ 中进行

Figure 2 (A) Absorption spectra of TPA-X-Q $\left(10 \mu \mathrm{mol} \cdot \mathrm{L}^{-1}\right)$, TPA-X-OH $\left(10 \mu \mathrm{mol} \cdot \mathrm{L}^{-1}\right)$, TPA-X-Q upon reaction with NQO1 (the concentration of probe was $10 \mu \mathrm{mol} \cdot \mathrm{L}^{-1}$; that of $\mathrm{NQO} 1$ was $15 \mu \mathrm{g} \cdot \mathrm{mL}^{-1}$; the reaction time was $40 \mathrm{~min}$ ). (B) The HR mass spectrum of TPA-X-Q upon reaction with NQO1 (the concentration of probe was $10 \mu \mathrm{mol} \cdot \mathrm{L}^{-1}$; that of NQO1 was $15 \mu \mathrm{g} \cdot \mathrm{mL}^{-1}$; the reaction time was $10 \mathrm{~min}$ ). (C) Time-dependent absorption spectra $(0 \sim 40 \mathrm{~min})$ of the probe in the presence of NQO1 $\left(15 \mu \mathrm{g} \cdot \mathrm{mL}^{-1}\right)$. (D) Absorption spectra at $40 \mathrm{~min}$ of the probe $\left(10 \mu \mathrm{mol} \cdot \mathrm{L}^{-1}\right)$ in the presence of varied levels of NQO1 $(0 \sim 15$ $\left.\mu \mathrm{g} \bullet \mathrm{mL}^{-1}\right)$. These measurements were performed in PBS $(\mathrm{pH}=7.4$, containing $\operatorname{DMSO}(\varphi=1 \%)$ and $100 \mu \mathrm{mol} \cdot \mathrm{L}^{-1} \mathrm{NADH}$ cofactor $)$ at $37{ }^{\circ} \mathrm{C}$

\section{2 探针对 NQO1 的响应及选择性}

为了探究并表征探针 TPA-X-Q 对 NQO1 的响应能 力, 我们进行了一系列的测试实验.

首先, 为探究响应机理, 我们测试了探针和 NQO1 反应液的吸收光谱和 HRMS 图谱. 如图 $2 \mathrm{~A}$ 所示, 与纯 探针溶液相比，探针与 NQO1 反应后的反应液的吸收发 生了明显的红移和增强, 并且和生色团化合物溶液的吸 收曲线相似. 此外, 探针和 NQO1 反应液(非充分反应) 的 HRMS 图谱(图 2B)中显示的 919.4298 和 687.3205 两 个峰分别对应探针 TPA-X-Q 和反应产物(生色团化合物 TPA-X-OH). 此结果说明该探针能对 NQO1 产生响应并 生成相应的生色团化合物.

之后，我们配制了探针 TPA-X-Q 的 PBS 缓冲液 $(\mathrm{pH}$ $=7.4$, 含 $\operatorname{DMSO}(\varphi=1 \%)$ 和 $100 \mu \mathrm{mol} \cdot \mathrm{L}^{-1}$ 辅酶因子 $\mathrm{NADH})$, 加入 $\mathrm{NQO} 1\left(15 \mu \mathrm{g} \cdot \mathrm{mL}^{-1}\right)$ 在 $37{ }^{\circ} \mathrm{C}$ 下培养, 并在 培养期间定期测试记录吸收光谱的变化. 结果如图 $2 \mathrm{C}$ 所示, 随着培养时间增加, 样品溶液的吸收光谱整体红
移至 $710 \mathrm{~nm}$ 且增强(图 S14). 随后我们又配制了探针溶 液, 并加入不同浓度的 $\mathrm{NQO} 1\left(0 \sim 15 \mu \mathrm{g} \cdot \mathrm{mL}^{-1}\right)$ 在 $37{ }^{\circ} \mathrm{C}$ 下培养, 并在培养时间为 $40 \mathrm{~min}$ 时测试记录它们吸收光 谱的变化, 结果如图 2D 和图 S15 所示. 随着 NQO1 浓 度的增加, 溶液的吸收光谱逐渐红移且增强. 更重要的 是, 不同波长下的光声强度测试表明, 与 $\mathrm{NQO}$ 反应后 的探针溶液, 其光声信号强度, 在波长 $710 \mathrm{~nm}$ 处展现出 最大值(图 3A). 并且在 $710 \mathrm{~nm}$ 的激发下, 相对光声强度 (I)与 $\mathrm{NQO} 1$ 的浓度 $(\mathrm{C})$ 在 $0 \sim 15 \mu \mathrm{g} \cdot \mathrm{mL}^{-1}$ 的范围内表现出 良好的线性关系(图 3B), 线性方程为 $I=0.41124 C+$ $0.00848(R=0.996)$. 因此, 由公式 $L O D($ 检测限 $)=3 \times$ $S . D$. (空白标准差) $/ K$ (回归线斜率)计算可知, 该探针对 $\mathrm{NQO} 1$ 的检测限为 $0.193 \mu \mathrm{g} \cdot \mathrm{mL}^{-1}$. 这些结果表明该探针 可以对 NQO1 产生响应, 并实现了增强型的光声检测.

此外，我们考察了该探针对 NQO1 响应的选择性测 试. 如图 3C 和 3D 所示, 只有当 NQO1 存在时, 探针溶 液才会产生显著的光声信号变化. 此结果说明该探针对 NQO1 的检测具有良好的特异性和抗干扰性, 有助于观
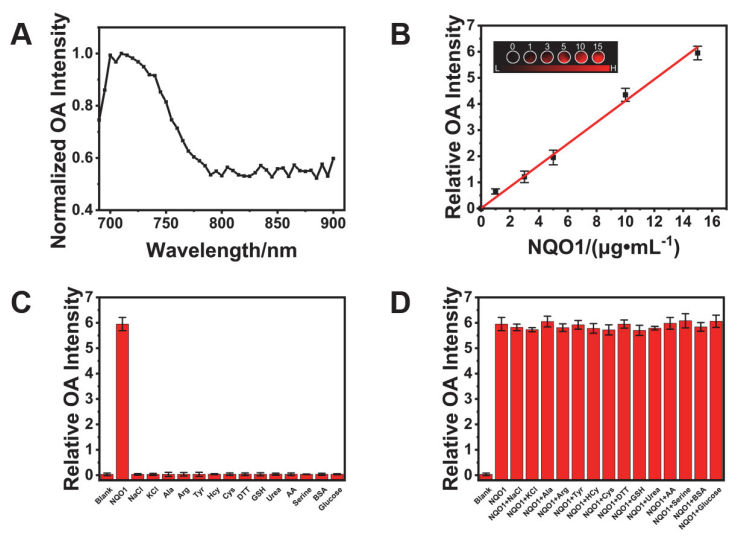

图 3 (A) $15 \mu \mathrm{g} \cdot \mathrm{mL}^{-1} \mathrm{NQO} 1$ 作用下探针 $\left(10 \mu \mathrm{mol} \cdot \mathrm{L}^{-1}\right)$ 的归一化光声强 度与波长关系图. (B) 探针 $\left(10 \mu \mathrm{mol} \cdot \mathrm{L}^{-1}\right)$ 对不同浓度 $\left(0 \sim 15 \mu \mathrm{g} \cdot \mathrm{mL}^{-1}\right)$ NQO1 的相对光声强度响应(样本量 $n=3$ ); 插图中为探针与不同浓度 NQO1 在假体中的光声图像(激发波长: $710 \mathrm{~nm})$. (C) 探针 $(10 \mu \mathrm{mol}$ • $\left.\mathrm{L}^{-1}\right)$ 对 $\mathrm{NQO1}\left(15 \mu \mathrm{g} \cdot \mathrm{mL}^{-1}\right)$ 及干扰物的光声响应, 及(D)抗干扰测试 $(n$ $=3)$; 测试浓度: 氯化钠 $(\mathrm{NaCl}) 、$ 氯化钾 $(\mathrm{KCl})$ 、丙氨酸 $(\mathrm{Ala}) 、$ 精氨酸 $(\mathrm{Arg})$ 、酪氨酸( Tyr $)$ 、高半胱氨酸 $(\mathrm{Hcy})$ 、半胱氨酸 $(\mathrm{Cys})$ 、二硫代苏糖 醇(DTT)、谷胱甘肽 $(\mathrm{GSH}) 、$ 尿素、抗坏血酸(AA)和丝氨酸为 $1 \mathrm{mmol}$ ・ $\mathrm{L}^{-1}$; 牛血清蛋白 $(\mathrm{BSA})$ 为 $20 \mathrm{mg} \cdot \mathrm{mL}^{-1}$; 葡萄糖为 $10 \mathrm{mmol} \cdot \mathrm{L}^{-1}$. 所有 测试均在 $37{ }^{\circ} \mathrm{C}$ 下的磷酸盐缓冲液 $(\mathrm{pH}=7.4$, 含 $\operatorname{DMSO}(\varphi=1 \%)$ 和 100 $\mu \mathrm{mol} \cdot \mathrm{L}^{-1}$ 辅酶因子 $\left.\mathrm{NADH}\right)$ 中进行

Figure 3 (A) The wavelength dependence of probe's $\left(10 \mu \mathrm{mol} \cdot \mathrm{L}^{-1}\right)$ normalized optoacoustic(OA) intensity in the presence of NQO1(15 $\left.\mu \mathrm{g} \cdot \mathrm{mL}^{-1}\right)$. (B) OA response of the probe $\left(10 \mu \mathrm{mol} \cdot \mathrm{L}^{-1}\right)$ to NQO1 of varied levels (sample size $n=3$ ); The inset shows the representative optoacoustic images of the probe in phantom at varied NQO1 level; Excitation wavelength: $710 \mathrm{~nm}$. (C) OA response of the probe $\left(10 \mu \mathrm{mol} \cdot \mathrm{L}^{-1}\right)$ in the presence of NQO1 $\left(15 \mu \mathrm{g} \cdot \mathrm{mL}^{-1}\right)$ and (D) anti-interference test $(n=3)$; The concentration of $\mathrm{NaCl}, \mathrm{KCl}$, alanine (Ala), arginine (Arg), tyrosine (Tyr), homocysteine (Hcy), cysteine (Cys), DL-dithiothreitol (DTT), glutathione (GSH), Urea, ascorbic acid (AA), serine was $1 \mathrm{mmol} \cdot \mathrm{L}^{-1}$; that of BSA was $20 \mathrm{mg} \cdot \mathrm{mL}^{-1}$; that of glucose was $10 \mathrm{mmol} \cdot \mathrm{L}^{-1}$. These measurements were performed in $\operatorname{PBS}(\mathrm{pH}=7.4$, containing $\operatorname{DMSO}(\varphi=$ $1 \%$ ) and $100 \mu \mathrm{mol} \cdot \mathrm{L}^{-1} \mathrm{NADH}$ cofactor) at $37{ }^{\circ} \mathrm{C}$ 
察生物体内的 $\mathrm{NQO} 1$ 在病理过程中含量的变化. 当探针 样品持续在紫外光照射下或在自然光下, 其在 $710 \mathrm{~nm}$ 处的吸收强度在 $1 \mathrm{~h}$ 的时间范围内没有明显变化(图 S16), 表明探针具有良好的光稳定性.

\section{3 乳腺癌小鼠模型光声成像}

在进行小鼠模型光声成像实验之前, 我们首先分别 用 MTT 法和小鼠体重变化来评估探针和生色团化合物 的细胞毒性和活体毒性. 实验结果显示(图 S17), 无论 是探针还是生色团化合物对 4T1 细胞系的细胞毒性基 本可忽略不计; 此外在注射探针和生色团化合物后, 小 鼠也并没有出现异常的体重变化(图 S18). 该结果表明 该探针体系具有良好的生物相容性和较低的生物毒性, 可用于活体成像.

为了评估该探针是否能对生物体内过量表达的 NQO1 产生响应, 从而实现乳腺肿瘤的成像, 我们建立 了乳腺癌小鼠模型. 为此, 分别向两组䧳性 BALB/c 裸 鼠的第四对乳腺脂肪垫注射等体积的 PBS 缓冲液 $(\mathrm{pH}=$ 7.4)和 $4 \mathrm{~T} 1$ 细胞悬浮液, 建立了对照组和小鼠乳腺癌模 型组. 如图 4A 所示, 一周后, 对照组小鼠的外形正常, 而模型组小鼠的第四对乳腺脂肪垫部位长出了明显的 肿瘤. 之后我们将 TPA-X-Q 探针注射入乳腺癌小鼠模

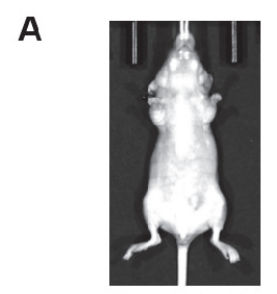

Control

B

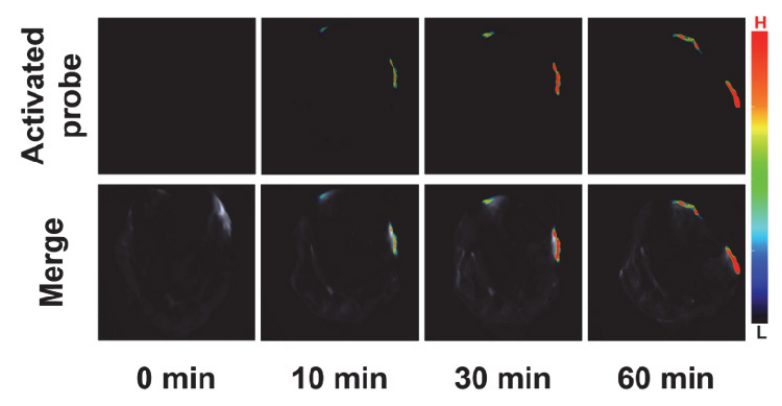

图 4 (A) 注射 PBS 缓冲液 (对照) 或含 $4 \mathrm{~T} 1$ 细胞悬浮缓冲液 (乳腺癌模 型) 1 周后小鼠的明场图像. (B) 乳腺癌小鼠模型在注射探针溶液之前 (0 min)和之后 10,30 和 $60 \mathrm{~min}$ 时的横截面光声图像; 右侧彩条代表不 同的 $\mathrm{OA}$ 信号强度. (上排: 激活探针的光声信号图; 下排: 激活探针 与灰度背景的光声信号叠加图)

Figure 4 (A) Representative bright-field images of nude mouse 1 week after injection of PBS (Control) or 4T1 cell suspension (Breast cancer model). (B) Representative cross-sectional OA images of the breast cancer-bearing nude mouse model before injection of the probe $(0 \mathrm{~min})$ and 10, 30, 60 min after injection of the probe; the color bar on the right side reflects different $\mathrm{OA}$ intensities. (Upper row: the OA signal image of activated probe; lower row: the overlay of the OA signal images of activated probe and grayscale background)
型的第四对乳腺脂肪垫, 并进行二维横截面光声成像和 正交视图三维光声成像. 如图 4B, 从左到右依次为探针 注射前, 注射后 10,30 和 $60 \mathrm{~min}$ 的二维光声截面图. 其 中上排为经过多光谱拆分后的激活探针 (即生色团 TPA-X-OH)所产生的信号图像, 下排为激活探针的信号 和灰度背景信号(主要来自血红蛋白、黑色素等内源性 光吸收剂等)的叠加图像. 从图中可以看出, 在注射探 针前 $(0 \mathrm{~min})$ 几乎无法观察到生色团的信号; 而注射探针 后 $10 \mathrm{~min}$, 肿瘤区域内开始产生微弱的光声信号, 并且 该信号在随后的 30 和 $60 \mathrm{~min}$ 明显增强.

此外，我们也通过 MSOT 的三维重建功能获得了正 交视图三维光声图像. 如图 5 所示, 从该图像中可看出, 不同时刻激活探针产生的光声信号及其在生物体内的 三维分布都可被清晰地观察到，因此肿瘤组织的分布状 况也可被确定. 这些结果证明, 探针 TPA-X-Q 可以在生 物体内对肿瘤组织中过量表达的 NQO1 进行增强型光 声响应进而产生明显光声信号, 并且具有提供高分辨 率、实时光声图像的能力. 不仅如此, 该探针还能通过 三维光声成像技术实现对乳腺肿瘤进行定位.

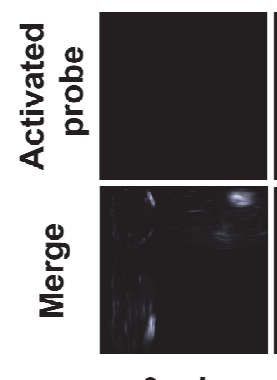

0 min

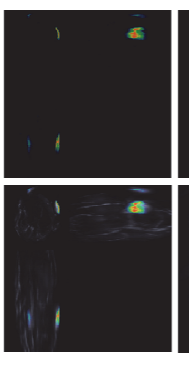

$10 \mathrm{~min}$

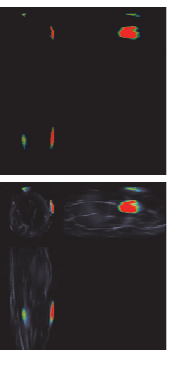

$30 \mathrm{~min}$

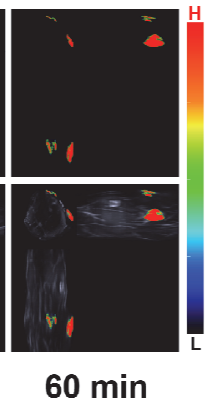

图 5 乳腺癌小鼠模型在注射探针溶液之前 $(0 \mathrm{~min})$ 和之后 10,30 和 $60 \mathrm{~min}$ 时的正交视图三维光声图像; 右侧彩条代表不同的 OA 信号强 度. (上排: 激活探针的光声信号图; 下排: 激活探针与灰度背景的光 声信号叠加图)

Figure 5 Representative 3D OA images of the breast cancer-bearing nude mouse model before injection of the probe $(0 \mathrm{~min})$ and 10, 30, 60 min after injection of the probe; the color bar on the right side reflects different OA intensities. (Upper row: the OA signal image of activated probe; lower row: the overlay of the OA signal images of activated probe and grayscale background)

\section{3 结论}

本项工作设计合成了一种新型的光声探针 TPA-X-Q. 该探针可对 NQO1 进行专一性的响应. 经 NQO1 的酶促反应，探针 TPA-X-Q生成相应的生色团化 合物 TPA-X-OH，进而产生较强的光声信号，因而可实 现对生物体内外 NQO1 的增强型光声检测与成像. 与近 些年报道的其它用于 NQO1 检测的荧光探针(表 S1)相 比, 该光声探针响应后的吸收波长较长 $(710 \mathrm{~nm})$, 有利 于减少光散射、降低背景干扰等. 此外, 改探针能在乳 腺癌小鼠模型的肿瘤病灶区域对过量表达的 NQO1 进 
行高分辨率、快速、实时的光声检测与成像, 并通过 MSOT 技术的三维成像功能实现对病灶区域的定位. 综 上所述, 本研究为重要生物标志物 NQO1 的检测提供了 一种手段, 而且该光声探针未来也有望应用于乳腺癌疾 病的早期诊断和乳腺癌病情发展的监测领域.

\section{4 实验部分}

\section{1 探针的制备}

探针的合成路线如图 S1 所示，具体合成步骤如下.

\subsection{1 化合物 2 的制备}

在氮气氛围下, 将 $30 \mathrm{~mL}$ 二甲基甲酰胺(DMF)置于 两口圆底烧瓶中. 在 $0{ }^{\circ} \mathrm{C}$ 下, 将 $20 \mathrm{~mL}$ 二氯甲烷和 20 $\mathrm{mL}$ 三氯氧磷(131 mmol)的混合液缓慢滴加入反应瓶中, 随后加热至室温搅拌 $60 \mathrm{~min}$. 再将 $5 \mathrm{~g}$ 环己酮 $(51 \mathrm{mmol}$ ) 缓慢加入反应瓶中. 加热至 $80{ }^{\circ} \mathrm{C}$ 回流反应 $2 \mathrm{~h}$. 反应结 束后, 将所得混合物倒入冰水中放置冰箱过夜. 过滤所 得悬浮液, 收集沉淀后真空干燥, 得到黄色粉末状固体 $(6.1 \mathrm{~g}, 69 \%)$ 化合物 $\mathbf{1}$.

将 $688 \mathrm{mg}$ 化合物 1 (4 mmol) 和 $2.4 \mathrm{~g}$ 的 1,2,3,3-四甲 基-3H-吲哚鎓碘化物( $8 \mathrm{mmol}$ )溶于 $30 \mathrm{~mL}$ 乙醇中并置于 单口圆底烧瓶, 随后加入 $656 \mathrm{mg}$ 乙醇钠 $(8 \mathrm{mmol})$. 在氮 气氛围下, 加热至 $80{ }^{\circ} \mathrm{C}$ 回流反应过夜. 所得反应液通 过真空旋转蒸发除去有机溶剂, 对剩余物通过柱层析的 方法纯化(固定相为硅胶, 流动相为二氯甲烷/甲醇, $V$ 二氯 甲烷 $: V$ 甲醇 $=25 ： 1)$, 得到绿色粉末状固体(1.66 g, 85\%) 化合物 2. ${ }^{1} \mathrm{H}$ NMR (600 MHz, Chloroform-d) $\delta 8.35$ (d, $J$ $=14.1 \mathrm{~Hz}, 2 \mathrm{H}), 7.39$ (t, $J=7.4 \mathrm{~Hz}, 4 \mathrm{H}), 7.25$ (t, $J=7.0$ $\mathrm{Hz}, 2 \mathrm{H}), 7.21(\mathrm{~d}, J=8.0 \mathrm{~Hz}, 2 \mathrm{H}), 6.22$ (d, $J=14.0 \mathrm{~Hz}$, 2H), $3.76(\mathrm{~s}, 6 \mathrm{H}), 2.75$ (t, $J=6.2 \mathrm{~Hz}, 4 \mathrm{H}), 1.95 \sim 2.0$ (m, $J$ $=6.4 \mathrm{~Hz}, 2 \mathrm{H}), 1.73(\mathrm{~s}, 12 \mathrm{H})$. HRMS (ESI): calcd. for $\mathrm{C}_{32} \mathrm{H}_{36} \mathrm{ClN}_{2}[\mathrm{M}]^{+} 483.2562$, found 483.2552.

\section{1 .2 化合物 3 的制备}

在氮气氛围下, 将 $54 \mathrm{mg}$ 的 5 -溴间苯二酚(0.29 $\mathrm{mmol}$ ) 和 $0.3 \mathrm{~mL}$ 三乙胺溶于 $4 \mathrm{~mL}$ 无水级 $\mathrm{DMF}$ 中并置 于单口烧瓶. 室温条件下搅拌 $10 \mathrm{~min}$. 将 $100 \mathrm{mg}$ 化合物 2(0.21 mmol)溶于 $2 \mathrm{~mL}$ 无水级 DMF 后获得混合溶液, 随后用注射器将该混合溶液缓慢加入到反应瓶中. 加热 至 $85{ }^{\circ} \mathrm{C}$ 反应 $3 \mathrm{~h}$. 所得反应液通过真空旋转蒸发除去有 机溶剂, 对剩余物通过柱层析的方法纯化(固定相为硅 胶, 流动相为二氯甲烷/甲醇, $V$ 二氯甲烷 $\left.: V_{\text {甲醇 }}=40: 1\right)$, 得 到蓝色粉末状固体(58 mg, 61\%)化合物 3. ${ }^{1} \mathrm{H}$ NMR (600 MHz, DMSO-d6) $\delta 9.71$ (s, 1H), 8.46 (d, $J=14.9 \mathrm{~Hz}, 1 \mathrm{H})$, $7.74(\mathrm{~d}, J=7.5 \mathrm{~Hz}, 1 \mathrm{H}), 7.64$ (d, $J=8.0 \mathrm{~Hz}, 1 \mathrm{H}), 7.53$ (t, $J$ $=7.8 \mathrm{~Hz}, 1 \mathrm{H}), 7.43(\mathrm{t}, J=7.4 \mathrm{~Hz}, 1 \mathrm{H}), 7.37(\mathrm{~s}, 1 \mathrm{H}), 7.05$ (s, 1H), $6.81(\mathrm{~s}, 1 \mathrm{H}), 6.51$ (d, J=14.9 Hz, 1H), 3.85 (s, $3 \mathrm{H}), 2.75$ (t, $J=6.2 \mathrm{~Hz}, 2 \mathrm{H}), 2.65$ (t, $J=6.3 \mathrm{~Hz}, 2 \mathrm{H})$, $1.89 \sim 1.79(\mathrm{~m}, 2 \mathrm{H}), 1.73(\mathrm{~s}, 6 \mathrm{H})$. HRMS (ESI): calcd. for $\mathrm{C}_{26} \mathrm{H}_{25} \mathrm{BrNO}_{2}[\mathrm{M}]^{+}$462.1063, found 462.1057.

\subsection{3 化合物 4(TPA-X-OH) 的制备}

在氮气氛围下, 将 $463 \mathrm{mg}$ 的化合物 $3(1 \mathrm{mmol}), 431$ $\mathrm{mg}$ 的 4-甲氧基- $N$-(4-甲氧基苯基)- $N$-(4-(4,4,5,5-四甲基1,3,2-二氧硼杂环戊烷-2-基)苯基)苯胺(1 mmol), $35 \mathrm{mg}$ 四(三苯基膦)钯 $(0.03 \mathrm{mmol})$ 和 $368 \mathrm{mg}$ 碳酸钾 $(2.7 \mathrm{mmol})$ 溶于 $8 \mathrm{~mL}$ 的 1,4-二氧六环中并置于单口圆底烧瓶. 加 热至 $80{ }^{\circ} \mathrm{C}$ 反应 $24 \mathrm{~h}$. 所得反应液用水与二氯甲烷进行 萃取 $\left(V_{\text {水 }}: V_{\text {二氯甲烷 }}=1: 1\right)$. 收集有机层, 并通过真空旋 转蒸发除去有机溶剂, 对剩余物通过柱层析的方法纯化 (固定相为硅胶, 流动相为二氯甲烷/甲醇, $V$ 二氯甲烷 $: V$ 甲醇 $=20 ： 1$ ), 得到绿色粉末状固体(495 mg, 72\%)化合物 4, 即光声生色团 TPA-X-OH. ${ }^{1} \mathrm{H}$ NMR (600 $\mathrm{MHz}$, DMSO- $\left.d_{6}\right) \delta 8.06(\mathrm{~d}, J=13.5 \mathrm{~Hz}, 1 \mathrm{H}), 7.63 \sim 7.62(\mathrm{~m}$, 2H), 7.47 (d, $J=7.4 \mathrm{~Hz}, 1 \mathrm{H}), 7.34$ (s, 1H), 7.31 (d, $J=7.7$ $\mathrm{Hz}, 1 \mathrm{H}), 7.20$ (d, $J=8.6 \mathrm{~Hz}, 2 \mathrm{H}), 7.12$ (d, $J=8.9 \mathrm{~Hz}, 4 \mathrm{H})$, $6.95(\mathrm{~d}, J=8.9 \mathrm{~Hz}, 4 \mathrm{H}), 6.80(\mathrm{~d}, J=8.2 \mathrm{~Hz}, 2 \mathrm{H}), 6.39$ (d, $J$ $=10.3 \mathrm{~Hz}, 1 \mathrm{H}), 6.32(\mathrm{~d}, J=10.6 \mathrm{~Hz}, 1 \mathrm{H}), 5.87$ (d, $J=13.4$ $\mathrm{Hz}, 1 \mathrm{H}), 3.76(\mathrm{~s}, 6 \mathrm{H}), 2.61 \sim 2.62(\mathrm{~m}, J=7.6 \mathrm{~Hz}, 2 \mathrm{H})$, $2.59 \sim 2.60(\mathrm{~m}, J=6.4 \mathrm{~Hz}, 2 \mathrm{H}), 1.76 \sim 1.78(\mathrm{~m}, J=6.8,6.4$ $\mathrm{Hz}, 2 \mathrm{H}), 1.65$ (s, 6H), 1.27 (s, 3H). ${ }^{13} \mathrm{C}$ NMR (151 MHz, Chloroform-d) $\delta 156.24,148.83,143.62,142.68,140.48$, $139.96,139.81,132.71,132.14,132.07,132.02,130.17$, $128.92,128.60,128.52,128.26,127.53,127.09,122.91$, $122.10,119.33,118.32,115.92,114.85,108.39,104.89$, 102.91, 55.53, 47.78, 29.95, 28.58, 24.89, 24.49, 21.26. HRMS (ESI): calcd. for $\mathrm{C}_{46} \mathrm{H}_{43} \mathrm{~N}_{2} \mathrm{O}_{4}[\mathrm{M}]^{+} 687.3217$, found 687.3207.

\subsection{4 化合物 5(TPA-X-Q) 的制备}

将 $344 \mathrm{mg}$ 的化合物 $4(0.5 \mathrm{mmol})$ 和 $125 \mathrm{mg}$ 的 3,3二甲基-3-(2,4,5-三甲基-3,6-二氧基环己-1,4-二烯-1-基) 丙酸 $(0.5 \mathrm{mmol})$ 溶于 $4 \mathrm{~mL}$ 二氯甲烷中并置于单口圆底 烧瓶, 冷却至 $0{ }^{\circ} \mathrm{C}$. 将 $207 \mathrm{mg}$ 二环己基碳二亚胺 (DCC)(1 mmol)和 $6 \mathrm{mg}$ 的 4-二甲氨基吡啶(DMAP)(0.05 $\mathrm{mmol})$ 溶于 $3 \mathrm{~mL}$ 二氯甲烷，配置成混合溶液，并在氮气 氛围下, 将该混合溶液用注射器缓慢滴加到反应瓶中. 加热至室温反应 $16 \mathrm{~h}$. 所得反应液用水与二氯甲烷进行 萃取 $\left(V_{\text {水 }}: V_{\text {二氯甲烷 }}=1: 1\right)$. 收集有机层, 并通过真空旋 转蒸发除去有机溶剂, 对剩余物通过柱层析的方法纯化 (固定相为硅胶, 流动相为二氯甲烷/甲醇, $V$ 二氯甲烷 $: V_{\text {甲醇 }}$ $=50: 1)$, 得到暗绿色粉末状固体(277 mg, 53\%)化合物 5, 即探针 TPA-X-Q. ${ }^{1} \mathrm{H}$ NMR (600 MHz, Chloroform-d) $\delta 8.62(\mathrm{~d}, J=15.1 \mathrm{~Hz}, 1 \mathrm{H}), 7.57 \sim 7.49(\mathrm{~m}, 3 \mathrm{H}), 7.44(\mathrm{t}, J$ $=7.3 \mathrm{~Hz}, 1 \mathrm{H}), 7.19(\mathrm{~s}, 1 \mathrm{H}), 7.14(\mathrm{~d}, \mathrm{~J}=8.8 \mathrm{~Hz}, 5 \mathrm{H}), 7.10$ $(\mathrm{d}, J=8.6 \mathrm{~Hz}, 2 \mathrm{H}), 6.97(\mathrm{~d}, J=8.6 \mathrm{~Hz}, 2 \mathrm{H}), 6.90 \sim 6.88$ $(\mathrm{m}, 5 \mathrm{H}), 6.86(\mathrm{~d}, J=2.2 \mathrm{~Hz}, 1 \mathrm{H}), 3.82(\mathrm{~s}, 6 \mathrm{H}), 3.32(\mathrm{~s}$, $2 \mathrm{H}), 2.85(\mathrm{~s}, 2 \mathrm{H}), 2.68 \sim 2.65(\mathrm{~m}, 2 \mathrm{H}), 2.60(\mathrm{~s}, 2 \mathrm{H}), 2.21$ $(\mathrm{s}, 3 \mathrm{H}), 1.99 \sim 1.89(\mathrm{~m}, 9 \mathrm{H}), 1.81(\mathrm{~s}, 6 \mathrm{H}), 1.56(\mathrm{~s}, 6 \mathrm{H}) .{ }^{13} \mathrm{C}$ NMR (151 MHz, Chloroform-d) $\delta$ 186.28, 177.52, 169.89, 
$158.68,155.48,155.27,152.72,150.62,148.27,145.08$, $141.10,140.84,139.07,138.72,129.29,129.13,128.83$, $128.75,128.38,126.81,126.24,126.09,121.19,118.69$, $118.34,118.04,116.74,114.57,113.90,113.84,112.35$, $106.59,105.77,54.51,49.77,46.67,37.51,33.59,28.68$, 28.10, 27.03, 23.54, 21.67, 19.26, 13.49, 11.66, 11.21 . HR-MS (ESI): calcd. for $\mathrm{C}_{60} \mathrm{H}_{59} \mathrm{~N}_{2} \mathrm{O}_{7}[\mathrm{M}]^{+} 919.4317$, found 919.4304.

\section{2 光谱分析}

将探针 TPA-X-Q 溶解至 DMSO 中配得浓度为 1 $\mathrm{mmol} \cdot \mathrm{L}^{-1}$ 的母液. 使用时, 随后将母液用 $\mathrm{PBS}$ 缓冲液 $(\mathrm{pH}=7.4)$ 稀释. 在探针对 NQO1 的体外响应测试中, 相 应浓度的 NQO1 加入到 TPA-X-Q 的 PBS 缓冲液 $(\mathrm{pH}=$ 7.4, TPA-X-Q 最终浓度 $10 \mu \mathrm{mol} \cdot \mathrm{L}^{-1}$, 含 $\operatorname{DMSO}(\varphi=1 \%)$ 和 $100 \mu \mathrm{mol} \cdot \mathrm{L}^{-1}$ 的辅酶因子 $\mathrm{NADH}$ )中. 于 $37{ }^{\circ} \mathrm{C}$ 水浴下 反应, 吸收光谱和相对光声强度光谱在期间定期测试获 得.

\section{3 细胞毒性实验}

探针 TPA-X-Q 和生色团化合物 TPA-X-OH 对细胞 的毒性由 MTT(ISO 10993-5 标准)法评估. 将 4T1 细胞 在含有 $10 \%$ 的 FBS 的培养基中培养后, 以 $1 \times 10^{5}$ cells $/ \mathrm{mL}$ 的浓度接种至 96 孔板中, 放入 $37{ }^{\circ} \mathrm{C}$ 含 $5 \%$ 的 $\mathrm{CO}_{2}$ 培养箱中培养. $24 \mathrm{~h}$ 后, 将其取出并移除培养基, 用 预热过的 PBS 缓冲液洗涤细胞, 之后用含有不同浓度 $\left(0 \sim 50 \mu \mathrm{mol} \cdot \mathrm{L}^{-1}\right) \mathrm{TPA}-\mathrm{X}-\mathrm{Q}$ 或 TPA-X-OH 的培养基 $\left(\varphi_{\mathrm{DMSO}}=0.2 \%\right)$ 继续培养 $24 \mathrm{~h}$. 之后用 $\mathrm{PBS}$ 缓冲液洗涤, 并加入含 $(0.5 \mathrm{mg} / \mathrm{mL})$ MTT 的培养基培养 $3 \mathrm{~h}$. 随后移除 培养基并每孔加入 $150 \mu \mathrm{L}$ 的 DMSO 溶解沉淀物. 最后 使用 Thermo MK3 ELISA 酶标仪测量 $570 \mathrm{~nm}$ 处的吸光 度. 每个浓度设置了 8 组测试, 细胞活性通过平均值和 标准偏差表征.

\section{4 生物安全性实验}

将雌性 BALB/c 裸鼠(6 周龄)随机分成三组, 每组 6 只. 分别将 $3.4 \mathrm{mg} / \mathrm{kg}$ 探针 TPA-X-Q 和 $4.6 \mathrm{mg} / \mathrm{kg}$ 生色 团化合物 TPA-X-OH 注射入其中两组小鼠内, 并将等体 积的 PBS 缓冲液 $(\mathrm{pH}=7.4)$ 注射入剩下一组小鼠内, 随 后连续 7 天测量和记录小鼠体重变化.

\section{5 乳腺癌小鼠模型的建立}

将雌性 BALB/c 裸鼠(6 周龄)随机分成两组, 每组 6 只. 将 $1 \times 10^{6}$ 个 $4 \mathrm{~T} 1$ 细胞悬浮于 $80 \mu \mathrm{L}$ PBS 缓冲液 $(\mathrm{pH}$ $=7.4)$ 中, 随后将该细胞悬浮液注射入其中一组小鼠的 第 4 对乳腺脂肪垫(每个脂肪垫注射 $80 \mu \mathrm{L}$ ), 作为乳腺癌 模型组; 并将等体积的 PBS 缓冲液注射入另一组小鼠体 内的相同位置, 作为对照组. 之后追踪并对比观察两组 小鼠的肿瘤发展情况.

\section{6 探针 TPA-X-Q 对小鼠乳腺癌区域的光声成像}

在进行活体光声成像实验之前, 通过呼吸罩使小鼠 (仰卧的姿势于 $34{ }^{\circ} \mathrm{C}$ 的成像腔内)吸入含有 $1 \%$ 异氟烷的 氧气进入麻醉状态进行成像. 将 $2.85 \mathrm{mg} / \mathrm{kg}$ 探针 TPA-X-Q 通过皮下注射的方式输入进小鼠第四对乳腺 脂肪垫, 然后进行成像. 分别在探针注射前 $(0 \mathrm{~min})$ 和注 射后 10, 30, $60 \mathrm{~min}$ 采集光声图像, 扫描步长为 $0.1 \mathrm{~mm}$. 根据生色团化合物 TPA-X-OH 和血红蛋白吸收光谱的 情况确定成像波长为 $680,690,700,710,730,760,800$ 和 $850 \mathrm{~nm}$ (作为背景). 每个波长记录 5 帧图像. 得到截面 图像后, 使用 MSOT 系统进行重建, 并得到三维图像; 最后利用光谱拆分获得来自生色团化合物 TPA-X-OH (激活探针)的光声信号和内源性光吸收剂(如血红蛋白、 去氧血红蛋白等)的光声信号.

\section{References}

[1] Asher, G.; Reuven, N.; Shaul, Y. BioEssays 2006, 28, 844.

[2] Clavería-Gimeno, R.; Velazquez-Campoy, A.; Pey, A. L. Arch. Biochem. Biophys. 2017, 636, 17.

[3] Pey, A. L. Int. J. Biol. Macromol. 2019, 126, 1223.

[4] Zhao, Z.; Li, X.; Wu, B.; Zhou, Y. Chin. J. Chem. 2020, 38, 714.

[5] Wang, N.; Zhao, J. Chin. J. Org. Chem. 2006, 26, 775 (in Chinese). (王乃兴, 赵嘉, 有机化学, 2006, 26, 775.)

[6] Mizumoto, A.; Ohashi, S.; Kamada, M.; Saito, T.; Nakai, Y.; Baba, K.; Hirohashi, K.; Mitani, Y.; Kikuchi, O.; Matsubara, J.; Yamada, A.; Takahashi, T.; Lee, H.; Okuno, Y.; Kanai, M.; Muto, M. J. Gastroenterol 2019, 54, 687.

[7] Luo, S.; Lei, K.; Xiang, D.; Ye, K. Oxid. Med. Cell. Longevity 2018, 2018,1 .

[8] Sies, H.; Berndt, C.; Jones, D. P. Annu. Rev. Biochem. 2017, 86 , 715 .

[9] Sabharwal, S. S.; Schumacker, P. T. Nat. Rev. Cancer 2014, 14, 709.

[10] Hayes, J. D.; Dinkova-Kostova, A. T.; Tew, K. D. Cancer Cell 2020, $38,167$.

[11] Sharma, C.; Wang, H.-X.; Li, Q.; Knoblich, K.; Reisenbichler, E. S.; Richardson, A. L.; Hemler, M. E. Cancer Res. 2017, 77, 6880.

[12] Danson, S.; Ward, T. H.; Butler, J.; Ranson, M. Cancer Treat. Rev. 2004, 30, 437.

[13] Dinkova-Kostova, A. T.; Talalay, P. Arch. Biochem. Biophys. 2010, $501,116$.

[14] Li, Z.; Zhang, Y.; Jin, T.; Men, J.; Lin, Z.; Qi, P.; Piao, Y.; Yan, G. BMC Cancer 2015, 15, 207

[15] Li, L.-S.; Reddy, S.; Lin, Z.-H.; Liu, S.; Park, H.; Chun, S. G.; Bornmann, W. G.; Thibodeaux, J.; Yan, J.; Chakrabarti, G.; Xie, X.; Sumer, B. D.; Boothman, D. A.; Yordy, J. S. Mol. Cancer Ther. 2016, 15, 1757 .

[16] Pani, G.; Giannoni, E.; Galeotti, T.; Chiarugi, P. Antioxid. Redox Signaling 2009, 11, 2791.

[17] Yang, Y.; Zhang, Y.; Wu, Q.; Cui, X.; Lin, Z.; Liu, S.; Chen, L. J. Exp. Clin. Cancer Res. 2014, 33, 14.

[18] Fagerholm, R.; Hofstetter, B.; Tommiska, J.; Aaltonen, K.; Vrtel, R.; Syrjäkoski, K.; Kallioniemi, A.; Kilpivaara, O.; Mannermaa, A.; Kosma, V.-M.; Uusitupa, M.; Eskelinen, M.; Kataja, V.; Aittomäki, K.; von Smitten, K.; Heikkilä, P.; Lukas, J.; Holli, K.; Bartkova, J.; Blomqvist, C.; Bartek, J.; Nevanlinna, H. Nat. Genet. 2008, 40, 844.

[19] Jamshidi, M.; Bartkova, J.; Greco, D.; Tommiska, J.; Fagerholm, R.; Aittomäki, K.; Mattson, J.; Villman, K.; Vrtel, R.; Lukas, J.; Heikkilä, P.; Blomqvist, C.; Bartek, J.; Nevanlinna, H. Breast Cancer Res. Treat. 2012, 132, 955.

[20] Qi, Y.; Huang, Y.; Li, B.; Zeng, F.; Wu, S. Anal. Chem. 2018, 90, 1014.

[21] Xiong, L.; Fan, Y.; Zhang, F. Acta Chim. Sinica 2019, 77, 1239 (in Chinese). (熊麟, 凡勇, 张凡, 化学学报, 2019, 77, 1239.)

[22] Niu, H.; Tang, J.; Zhu, X.; Li, Z.; Zhang, Y.; Ye, Y.; Zhao, Y. Chem. Commun. 2020, 56, 7710

[23] Specht, E. A.; Braselmann, E.; Palmer, A. E. Annu. Rev. Physiol. 
2017, 79, 93

[24] Wu, Y.; Wang, J.; Zeng, F.; Huang, S.; Huang, J.; Xie, H.; Yu, C.; Wu, S. ACS Appl. Mater. Interfaces 2016, 8, 1511.

[25] Chang, Y.; Li, B.; Guo, M.; Cai, Y.; Xu, K. Chin. J. Org. Chem. 2019, 39, 2485 (in Chinese). (常永新, 李白, 郭沝, 蔡永红, 徐括 喜, 有机化学, 2019, 39, 2485.)

[26] Christensen, P. R.; Wolf, M. O. Adv. Funct. Mater. 2016, 26, 8471.

[27] Wang, J.; Wu, Y.; Sun, L.; Zeng, F.; Wu, S. Acta Chim. Sinica 2016, 74,910 (in Chinese). (王俊, 武英龙, 孙立和, 曾钫, 吴水珠, 化 学学报, 2016, 74,910.)

[28] Liu, P.; Xu, J.; Yan, D.; Zhang, P.; Zeng, F.; Li, B.; Wu, S. Chem. Commun. 2015, 51,9567.

[29] Yang, Q.; Wen, Y.; Xu, J.; Shao, S. Talanta 2020, 216, 120982.

[30] Yuan, Z.; Xu, M.; Wu, T.; Zhang, X.; Shen, Y.; Ernest, U.; Gui, L.; Wang, F.; He, Q.; Chen, H. Talanta 2019, 198, 323

[31] Shen, Z.; Prasai, B.; Nakamura, Y.; Kobayashi, H.; Jackson, M. S.; McCarley, R. L. ACS Chem. Biol. 2017, 12, 1121.

[32] Hettiarachchi, S. U.; Prasai, B.; McCarley, R. L. J. Am. Chem. Soc. 2014, 136, 7575 .

[33] Pan, D.; Luo, F.; Liu, X.; Liu, W.; Chen, W.; Liu, F.; Kuang, Y.-Q.; Jiang, J.-H. Analyst 2017, 142, 2624.

[34] Cheng, Z.; Valença, W. O.; Dias, G. G.; Scott, J.; Barth, N. D.; de Moliner, F.; Souza, G. B. P.; Mellanby, R. J.; Vendrell, M.; da Silva Júnior, E. N. Bioorg. Med. Chem. 2019, 27, 3938.

[35] Ovsepian, S. V.; Olefir, I.; Westmeyer, G.; Razansky, D.; Ntziachristos, V. Neuron 2017, 96, 966

[36] Huang, J.; Wu, Y.; Zeng, F.; Wu, S. Theranostics 2019, 9, 7313.
[37] Ouyang, J.; Sun, L.; Zeng, Z.; Zeng, C.; Zeng, F.; Wu, S. Angew. Chem. Int. Ed. Eng. 2020, 59, 10111.

[38] Reber, J.; Willershäuser, M.; Karlas, A.; Paul-Yuan, K.; Diot, G.; Franz, D.; Fromme, T.; Ovsepian, S. V.; Bézière, N.; Dubikovskaya, E.; Karampinos, D. C.; Holzapfel, C.; Hauner, H.; Klingenspor, M.; Ntziachristos, V. Cell Metab. 2018, 27, 689.

[39] Zhan, C.; Huang, Y.; Lin, G.; Huang, S.; Zeng, F.; Wu, S. Small 2019, 15, 1900309.

[40] Wu, Y.; Chen, J.; Sun, L.; Zeng, F.; Wu, S. Adv. Funct. Mater. 2019, 29, 1807960 .

[41] Diot, G.; Metz, S.; Noske, A.; Liapis, E.; Schroeder, B.; Ovsepian, S. V.; Meier, R.; Rummeny, E.; Ntziachristos, V. Clin. Cancer Res. 2017, 23, 6912 .

[42] Ntziachristos, V; Razansky, D. Chem. Rev. 2010, 110, 2783.

[43] Luís Dean-Ben, X.; Razansky, D. Light: Sci. Appl. 2018, 7, 18004.

[44] Gujrati, V.; Mishra, A.; Ntziachristos, V. Chem. Commun. 2017, 53, 4653.

[45] Schellenberg, M. W.; Hunt, H. K. Photoacoustics 2018, 11, 14.

[46] Song, X.; Bian, H.; Wang, C.; Hu, M.; Li, N.; Xiao, Y. Org. Biomol. Chem. 2017, 15, 8091 .

[47] Kim, H.; Lee, M.; Kim, H.; Kim, S.; Yoon, J. Chem. Soc. Rev. 2008, 37,1465

[48] Han, J.; Burgess, K.; Chem. Rev. 2010, 110, 2709.

[49] Mendoza, M. F.; Hollabaugh, N. M.; Hettiarachchi, S. U.; McCarley, R. L. Biochemistry 2012, 51, 8014.

[50] Wu, Y.; Huang, S.; Wang, J.; Sun, L.; Zeng, F.; Wu, S. Nat. Commun. 2018, 9, 3983.

(Yang, X.) 